\title{
Author Index Vol. 63, 1996
}

Abe,K. 42 Abe,S. 106,381 Acican, T. 25 Ahmad, H.R. 288 Aizawa, H. 205 Akino,T. 381

Alamé,T. 388 Alejo,M. 59 Alfaro,A. 403 Alifano,M. 292 Allegra,L. 61, 174 Alsina,J.M. 160

Altmeyer, P. 368 Altose,M.D. 137 Amour, A. 277 Andersson, S.E. 111 Annesi, I. 352 Aparicio, J. 251 Arborelius, M. 66 Arias, M. 59 Arnaud,A. 100 Arosio, C. 61 Arribas, J.M. 199 Aydin,A. 25

Bagci Ceyhan, B. 17 Ballabio,P. 28 Bambery, P. 52 Bando,T. 309 Banerjee, A.K. 52 Barck,C. 325 Barrière, J.-R. 100 Bauer, T. 164 Baumann, G. 195 Baur,X. 84, 123,368 Berkman,N. 391 Betsuyaku, T. 333 Bianchi, L. 28 Bignamini, A. 28 Blasi,F. 61 Bogaard, J.M. 1 Boni,E. 8 Borer, H. 400 Boulet, L.-P. 304 Brugués, J. 59

Carratù,L. 292 Cases, E. 241 Castelao-Naval, J. 339 Castriotta, R.J. 283 Cebrián, J. 73

Çelikel,T. 17 Cherniack, N.S. 137

Chin,K. 223 Coll,R. 230 Compte, L. 73 Conrad, K. 368 Cordaro, C.I. 174 Cordero, P. 241

Cosentini, R. 61

Dahl,M. 325 Dartevelle, P.G. 49 Dazzi,H. 117 Degens, P. 123,368 Delgado,E. 120 Deliz,R. 246

DelPezzo,M. 292 Demiralp, E. 17 Demirel,Y.S. 25 Deodhar, S.D. 52 DeVito,E.L. 187

Dicroco,A. 100 Diego, A. de 73 Domingo, C. 230 Domingo, E. 230 Dulmet,E.M. 49

Eklund,A. 325 Emslander, H.-P. 195 Escudero,C. 199 Ewert,R. 195 Ewig, S. 164

Ferrandis, S. 73 Filippi,B. 8 Fité,E. 160 Fogue, L. 120 Frey,M. 400 Fujii,T. 236 Fujimura, M. 309 Fujimura, S. 191 Fujishima, T. 381 Fujita,A. 106 Fujita,M. 223

Galán,G. 241 Garcia-Barreto, L. 246 García-García, J.M. 199 Garewal,G. 52 Gäumann, N. 117 Ge,R.-L. 346 Gelmetti,W. 181 Ghio,A.J. 254 GilSuay,V. 267 Gómez-Aldaraví, L. 251

Goto,H. 78 Grassi,C. 174 Grassi,G. 28 Grassi,G.G. 28 Grassi,V. 8 Guignon, I. 100 Gürbüz, L. 25

Hamabe, S. 35 Hanson, B.S. 66 Hara,K. 42 Hara,N. 205 Hashimoto, T. 397 Hasper, E. 164 Hasumi, T. 191 Hedblad,B. 66 Heitmann, R. 123 Hemsén, A. 111 Hernandez, J.R. 199 Heudes, D. 277 Hiepe, F. 195 Hillenbach, C. 123 Hirasawa,M. 106,381 Hirata, K. 236 Honda, Y. 381 Horovitz, O. 261 Horowitz, S. 261

Ieki,R. 78 Igarashi, T. 106 Iguchi,M. 78 Ikeda,S. 35 Imai,T. 397 Inoue, H. 205 Ishida,T. 314 Ishimura, T. 223 Isimer, A. 25 Israel, R.H. 55 Izquierdo, J. 230 Izquierdo-Alonso, J.L. 339 Janzon, L. 66 Jiva,T.M. 55 Johard,U. 325 John,M. 195

Juretschke-Moragues, M.A. 339

Kadota, J.-i. 42 Kakkar,N. 52 Kanazawa, H. 236 Kawahara, E. 397 Kawakami, Y. 333

Keller,K. 388 Keller, R. 400 Khan, M.A. 288 Kido,M. 94 Klamburg,J. 230 Klockars,M. 272

Klose,K.J. 181 Kodama,T. 106 Köhler,D. 155 Kohno, S. 42 Kondo,T. 191 Konrádová, V. 150

Korobaeff,M. 352 Koto,H. 205 Krajina,A. 394 Kramer, M.R. 391 Kubini,R. 164 Kudoh,S. 236

Kuno,K. 223 Kurihara,N. 236 Kuroki,Y. 381

Laprise, C. 304 Leon, M. 73 Liard,R. 352 Lieberman, D. 261 Lin,W.H. 144 Liss,H. 391

Llorca,C. 251 Loukides, S. 170 Lüderitz, B. 164 Lundberg, J.M. 111 Lunteren, E. van 88 
Maccario, J. 352 Macchiarini, P. 49 Malerba,M. 8 Mañá,J. 160,298 Mangiarotti, P. 28 Manresa, F. 298 Marco, V. 267 Marek,W. 123 Marklein,G. 164 Marrón,M.G. 199 Martin, H. 195

Martinez, E. 267 Martinez, J. 199 Martínez-Moragón, E. 251 Martínez-Muñiz, M.A. 199

Maruyama, N. 358 Maruyama, R. 314 Masuda,L. 358 Matsuda,L. 309

Matsumoto, K. 205 Matthys,H. 321 Mehlhorn,J. 368 Meloni,F. 28 Menéndez, R. 267 Metersky, M.L. 283 Micco,A. 292 Michel, O. 388 Miller, MJ. 88 Mínguez, S. 312 Misirhgil, Z. 25

Mitsudomi, T. 314 Miyahara, Y. 35 Miyamoto, K. 333 Monte, C. 199 Montiel,G.C. 187

Montpréville, V.T. de 49 Montserrat, J.M. 312 Morales, P. 241 Morera,J. 160,230 Morice,A.H.

217,376 Mormile, A.F. 292 Mormile,M. 292 Mungan, D. 25

Nagata, N. 94 Naito,T. 35 Nara,M. 358 Nauffal,D. 267 Navrátil,P. 394 Ness, V. 363

Neukirch,F. 352 Nikaido,Y. 94 Nishimura, M. 333 Nishioka,K. 314 Noda,Y. 309 Nyberg,P.

272

Odeh,M. 213 Ohi,M. 223 Ohta,G. 309 Ohta,M. 35 Okamura, T. 78 Oku,Y. 137 Oliven,A. 213

Oltra,A. 251

Ortiz-Saracho, J. 120 Otsuka,T. 236

Panos,R.J. 346 Paris, F. 241 Parkinson, D. 144 Pastor, J. 241 Pastor, M. 251 Pedro-Botet, J. 312

Perez-Rodriguez, E. 120 Perpiñá, M. 73,403 Pettersson, T. 272 Philip-Joet, F. 100 Piantadosi,

C.A. 254 Piqueras, A. 241 Plaza, V. 59 Poe,R.H. 55 Pohle, W.R. von 318 Polyzogopoulos, D.

170 Porath,A. 261 Porcel,G. 131 Pracyk,J.B. 254 Pujol, R. 298

Quadrelli, S.A. 131, 187

Raccanelli, R. 61 Ramos-Martos, A. 339 Randazzo, A. 61 Rausch,V. 123 Rayani,H.H. 288

Reboud-Ravaux, M. 277 Resl,M. 394 Rey,M. 100 Reynaud,M. 100 Rihs,H.-P. 368 Riska,H.

272 Rodriguez, W. 246 Rodríguez-Glez Moro, J.M.

339 Roggli,V.L. 254 Roig,J. 230 Romagnoni, G. 8 Roncoroni,A.J. 131, 187 Rosberg, H.-E. 66

Rossi, S. 61

Ruiz-Manzano, J. 230 Russi,E.W. 117

Sagawa, M. 191 Saidel,G.M. 137 Saitoh, G. 314 Sakai,A. 346 Sakito,O. 42 Salazar,A. 298

Sánchez-Antuña, A. 199 Sánchez-Hernández, I. 339 Sasaki, H. 106 Sayal,A. 25 Schlaeffer, F.

261 Schnall,R.P. 213 Schönhofer, B. 155 Ségala,C. 352 Sergysels,R. 388 Serra-Batlles, J. 59

Sheedy,W. 217,376 Shijubo,N. 106,381 Shimizu,J. 397 Shimura, S. 358 Shirai,R. 42 Shirato,K. 358 Simonson, S.G. 254 Sin,B. 25 Sköld,C.M. 325 Sluys, H.C.M. van der 1 Smaoui, H. 277 Söderblom, T. 272 Sofia, M. 292 Soler,J.J. 403 Stewart, A.G. 376 Strom, K. 66 Sugimachi, K. 314 Sugita,M. 191 Supervía, A. 312

Takahashi, H. 381 Takata,S. 205 Takayama, K. 94 Takeda,T. 236 Takenaka,K. 314 Takeoka,M. 346 Tanaka, S. 236

Taniguchi, S. 346 Tantucci, C. 8 Tarsia, P. 61 Tek$^{3} / 8$ en, F. 25 Thompson, J.S. 217 Thrall, R.S. 144 Turcotte, J.G. 144 Tuyet,J. 312

Ueda,G. 346 Uhlík,J. 150 Ulmer,W.T. 123

Vafaie,H. 88 Vallés,A. 59 Van der Meché, F.G.A. 1 Vasishta, R.K. 52 Vega,W. 246 Vellani, C.W. 288 Verbraak, A.F.M. 1 Ververs, C.C.M. 1 Vidal,R. 120 Voshaar, T. 155

Wagner, U. 181 Wakabayashi, A. 223 Walthers, E.M. 181 Weber, K. 368 Wichert, P. von 181

Wiebe,V. 368 Witt,C. 195

Yamaguchi, K. 106 Yokosaki,Y. 94 Yoshioka, A. 333 Young, S.L. 254 Yuasa,K. 78

Zackrisson, C. 111 Zaidi, S.R. 288 Zajícová, A. 150 Zinman, R. 363 Zocová, J. 150 
Author Index Vol. 63, 1996 